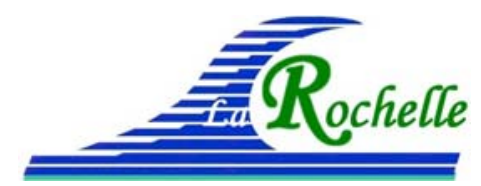

XVèmes Journées Nationales Génie Côtier - Génie Civil

La Rochelle, 29 au 31 mai 2018

DOI:10.5150/jngcgc.2018.013 ～(C) Editions Paralia CFL

disponible en ligne - http://www.paralia.fr - available online

\title{
Étude comparative des méthodes de reconstruction du champ de vagues à partir de la mesure de pression : Application à la plage de la Salie
}

\section{Arthur MOURAGUES ${ }^{1}$, Philippe BONNETON ${ }^{1}$, David LANNES ${ }^{2}$, Vincent MARIEU ${ }^{1}$, Guillaume DETANDT ${ }^{1}$, Bruno CASTELLE ${ }^{1}$, Pierre-Antoine PONCET $^{3}$, Benjamin DUBARBIER ${ }^{1}$}

1. Université de Bordeaux, UMR 5805 CNRS-EPOC, France

2. Université de Bordeaux, UMR 5251 CNRS-IMB, France

3. Université de Pau et des Pays de l'Adour, France

\section{Résumé :}

La reconstruction du champ de vagues basée sur la théorie linéaire, communément appelée méthode de la fonction de transfert, décrit assez bien les caractéristiques moyennes des vagues. Elle est couramment utilisée pour déterminer les paramètres statistiques des vagues mais sous-estime la hauteur des plus grosses vagues. Cependant, bien estimer ces vagues énergétiques est primordial pour l'étude des risques côtiers lors d'évènements extrêmes. La reconstruction linéaire nécessite l'application d'une fréquence de coupure du spectre d'énergie qui induit une perte d'information. Des techniques empiriques qui essaient de combler artificiellement le spectre d'énergie n'améliorent que très peu les plus grosses vagues. Récemment, des formules simples à implémenter permettant des reconstructions linéaires et non-linéaires ont été développées.

Nous présentons et comparons dans cet article différentes méthodes de reconstruction du champ de vagues à partir de la pression mesurée au fond lors d'une campagne de mesure réalisée sur la plage de La Salie en avril 2017. Notre étude est centrée sur des vagues très non-linéaires et faiblement dispersives. Une analyse vague à vague met en évidence la capacité de la reconstruction non-linéaire faiblement dispersive à reproduire la hauteur et l'asymétrie des plus grosses vagues contrairement à la formule linéaire couramment utilisée en ingénierie côtière.

Mots-clés: Théorie linéaire, non-linéarité, capteurs de pression, reconstruction du champ de vagues.

\section{Introduction}

Les capteurs de pression sont utilisés depuis longtemps pour leur robustesse, leur aspect bon marché et leur facilité de déploiement. Cependant, ils ne mesurent pas directement la surface libre des vagues. La reconstruction basée sur la théorie linéaire, communément appelée méthode de la fonction de transfert, est la méthode couramment utilisée en ingénierie côtière (GUZA \& THORNTON, 1980 ; TSAI et al., 2005) et 


\section{Thème 1 - Hydrodynamique côtière}

décrit assez bien les caractéristiques moyennes des vagues (BISHOP \& DONELAN, 1987). La reconstruction linéaire nécessite l'application d'une fréquence de coupure au spectre d'énergie induisant une perte d'information. Des techniques empiriques telles que celles de KARIMPOUR \& CHEN (2017) ou de NEUMEIER qui essaient de combler artificiellement le spectre d'énergie sont généralement appliquées. Néanmoins, en eau peu profonde, ces techniques ne permettent pas de bien reproduire l'amplitude des plus grosses vagues. MARTINS et al. (2017) ont montré que, avant le déferlement, la théorie linéaire pouvait sous-estimer jusqu'à 30\% la hauteur individuelle des vagues. Cependant, la mesure précise des vagues est capitale pour l'étude de la dynamique hydrosédimentaire en zone côtière. Par exemple, l'étude de l'impact des vagues de submersion nécessite de bien reproduire la hauteur des plus grosses vagues. La bonne description de l'asymétrie des vagues est essentielle pour l'analyse du transport sédimentaire (DUBARBIER et al., 2015).

BONNETON et al. (2018) ont développé des formules applicables localement en temps et permettant une reconstruction non-linéaire du champ de vagues en eau peu profonde. Nous appliquons ces méthodes de reconstruction aux données acquises dans le cadre d'une campagne de mesure sur la plage de La Salie en avril 2017. Après avoir présenté le cadre expérimental et les méthodes de reconstruction, nous effectuerons une étude comparative lorsque les vagues sont très non-linéaires.

\section{Mesures in situ}

\subsection{Site d'étude et instrumentation}

Du 13 au 14 avril 2017, une campagne de mesure a été effectuée sur la plage de La Salie (sud-ouest de la France) dont l'objectif était d'acquérir des données de pression et de surface libre pour des vagues se propageant en faible profondeur. Une structure de mouillage composée d'un capteur de pression relié à un profileur de courant acoustique Nortek Signature $1000 \mathrm{kHz}$ a été déployée à marée basse. Le mode AST (Acoustic Surface Tracking) du Signature permet de fournir une mesure directe de la surface libre. Les 2 capteurs ont une fréquence d'échantillonnage de $8 \mathrm{~Hz}$.

\subsection{Méthodes}

La mesure de la pression nous donne la profondeur d'eau hydrostatique $(\mathrm{H})$ définie par : $\mathrm{h}_{\mathrm{H}}=\zeta_{\mathrm{H}}+\mathrm{h}_{0}-\delta_{\mathrm{m}}=\frac{\mathrm{P}_{\mathrm{m}}-\mathrm{P}_{\mathrm{a}}}{\rho \mathrm{g}}$

où $\zeta_{\mathrm{H}}$ est la surface libre hydrostatique, $\mathrm{P}_{\mathrm{m}}$ la pression mesurée, $\mathrm{P}_{\mathrm{a}}$ la pression atmosphérique, $\rho$ la masse volumique, $h_{0}$ la profondeur moyenne et $\delta_{\mathrm{m}}=0,69 \mathrm{~m}$ la hauteur du capteur par rapport au fond.

Le signal acoustique est bruité (environ 3\% de valeurs aberrantes) à cause des bulles d'air qui peuvent gêner la propagation du signal sur la colonne d'eau. Pour supprimer 


\section{XVèmes Journées Nationales Génie Côtier - Génie Civil \\ La Rochelle, 29 au 31 mai 2018}

les valeurs aberrantes, on rejette celles étant deux fois supérieures à la hauteur hydrostatique. Puis, un critère sur la pente nous permet de supprimer les valeurs aberrantes restantes. Enfin, on y applique un filtre passe-bas $(1 \mathrm{~Hz})$ pour éliminer le bruit de la mesure. Le signal obtenu est la profondeur d'eau mesurée $h_{m}=\zeta_{m}+h_{0}-\delta_{m}$ où $\zeta_{m}$ est la surface libre mesurée. Dans la suite, on s'intéresse à une fenêtre de 10 minutes avant le déferlement lorsque les vagues sont très non-linéaires $\left(\varepsilon_{\max }=a_{\max } / h_{0}=0,3\right.$ où $a_{\max }$ est l'amplitude de la plus grosse vague et $\left.\mathrm{h}_{0}=2,25 \mathrm{~m}\right)$ et faiblement dispersives $(\mu$ $=\left(\mathrm{kh}_{0}\right)^{2}=0,07$ où $\mathrm{k}$ est le nombre d'onde). La non-linéarité de l'écoulement est illustrée par l'asymétrie des vagues (voir figure 1) et par la présence d'harmoniques secondaires bien développés dans le spectre d'énergie (voir figure 2). On peut noter la présence de mer de vent induisant des vagues courtes notamment dans le creux des plus grosses vagues (voir figure 1).

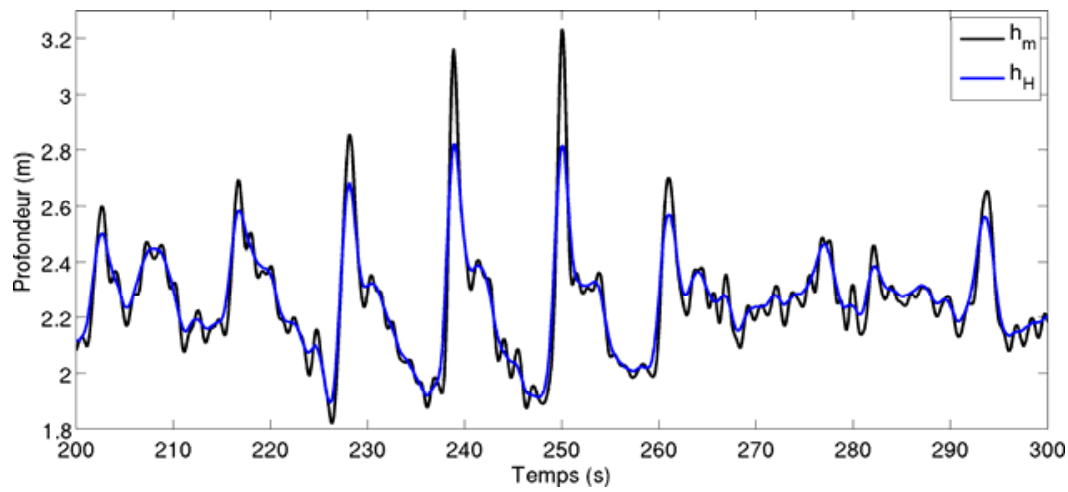

Figure 1. Profondeur d'eau mesurée $\left(h_{m}\right)$ et calculée par la reconstruction $H\left(h_{H}\right)$. Zoom sur un groupe de vagues.

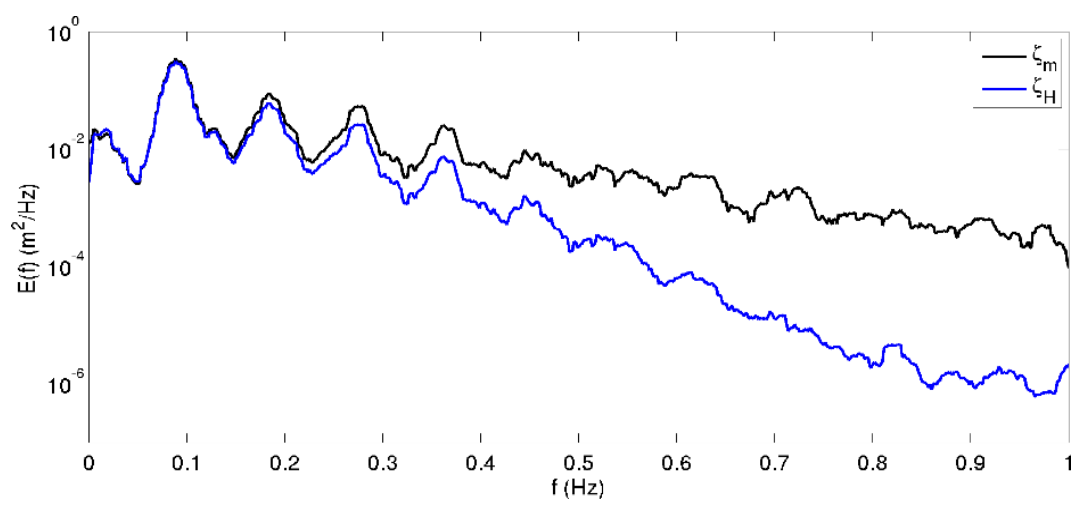

Figure 2. Spectres de densité d'énergie, $E(f)$, de la surface libre mesurée $\left(\zeta_{m}\right)$ et calculée par la reconstruction $H\left(\zeta_{H}\right)$. 


\section{Thème 1 - Hydrodynamique côtière}

\section{Méthodes de reconstruction}

\subsection{Reconstruction linéaire et fréquence de coupure}

La méthode de reconstruction la plus utilisée en ingénierie côtière est celle basée sur la théorie linéaire. Celle-ci s'écrit de la manière suivante :

$\tilde{\zeta}_{\mathrm{L}}=\mathrm{K}_{\mathrm{P}}(\omega) \tilde{\zeta}_{\mathrm{H}}$,

où : $\mathrm{K}_{\mathrm{P}}(\omega)=\frac{\cosh \left(\mathrm{k}(\omega) \mathrm{h}_{0}\right)}{\cosh \left(\mathrm{k}(\omega) \delta_{\mathrm{m}}\right)}$ est le facteur de correction non-hydrostatique, $\tilde{\zeta}_{\mathrm{L}}$ et $\tilde{\zeta}_{\mathrm{H}}$ sont les transformées de Fourier de la surface libre linéaire et hydrostatique, et $\omega$ la pulsation. $\mathrm{k}$ et $\omega$ sont reliés par la relation de dispersion suivante :

$\omega^{2}=g k \tanh \left(k h_{0}\right)$

En appliquant les équations (2) et (3) aux mesures in-situ, on remarque que l'énergie de la reconstruction linéaire ( $\zeta_{\mathrm{L}, \mathrm{PC}}$ sur la figure 3 ) diverge pour les hautes fréquences. Ce problème n'est pas lié au bruit de la mesure mais au fait que la relation de dispersion (3) induit une très forte erreur lorsque les vagues sont fortement non-linéaires.

Il est alors nécessaire d'introduire une fréquence de coupure $f_{c}$. Au-delà de cette fréquence, on suppose le spectre comme hydrostatique c'est-à-dire :

$K_{P}(\omega)= \begin{cases}\frac{\cosh \left(k(\omega) h_{0}\right)}{\cosh \left(k(\omega) \delta_{m}\right)}, & \frac{\omega}{2 \pi} \leq f_{c} \\ 1, & \frac{\omega}{2 \pi}>f_{c}\end{cases}$

L'équation (4) est la reconstruction linéaire avec une coupure brutale (LCB). De nombreuses méthodes peuvent être utilisées pour fixer $f_{c}$. Ces méthodes étant empiriques (comme celle de GREEN, 1999), nous choisissons de fixer $f_{c}$ en comparant les spectres d'énergie de $\zeta_{\mathrm{L}, \mathrm{PC}}$ et $\zeta_{\mathrm{m}}$ (voir figure 3). Nous coupons le spectre de la reconstruction linéaire dès que celui-ci diverge de celui de la mesure (en $f_{c}=0.42 \mathrm{~Hz}$ ). $\mathrm{La}$ fréquence de coupure induit donc une perte d'information dans les hautes fréquences. Pour pallier ce manque d'information, différentes techniques empiriques existent pour combler le spectre au-delà de $\mathrm{f}_{\mathrm{c}}$. Une des plus utilisées est la linéarisation de $K_{P}$ (NEUMEIER) telle que :

$K_{P}(\omega)=\left\{\begin{array}{lr}\frac{\cosh \left(k(\omega) h_{0}\right)}{\cosh \left(k(\omega) \delta_{m}\right)}, \quad \frac{\omega}{2 \pi} \leq f_{c} \\ K_{P, l i n}, \quad f_{\text {lin }}>\frac{\omega}{2 \pi}>f_{c}, \\ 1, & \frac{\omega}{2 \pi}>f_{\text {lin }}\end{array}\right.$

où $\mathrm{K}_{\mathrm{P}}$, lin et flin sont fixés arbitrairement. L'équation (5) est la reconstruction linéaire avec une coupure linéaire (LCL).

Une autre technique est de raccorder le spectre par un spectre de JONSWAP :

$E(f)=E\left(f_{c}\right)\left(\frac{f}{f_{c}}\right)^{-n}, \quad f>f_{c}$

où la valeur de $n$ est discutable. KARIMPOUR \& CHEN (2017) utilisent cette méthode. Même si ces techniques améliorent le calcul de la hauteur significative, elles ne permettent pas de reproduire la hauteur des plus grosses vagues ni d'entièrement éviter les oscillations parasites liées à la fréquence de coupure (voir figure 4 et tableau 1). 


\section{XVèmes Journées Nationales Génie Côtier - Génie Civil \\ La Rochelle, 29 au 31 mai 2018}

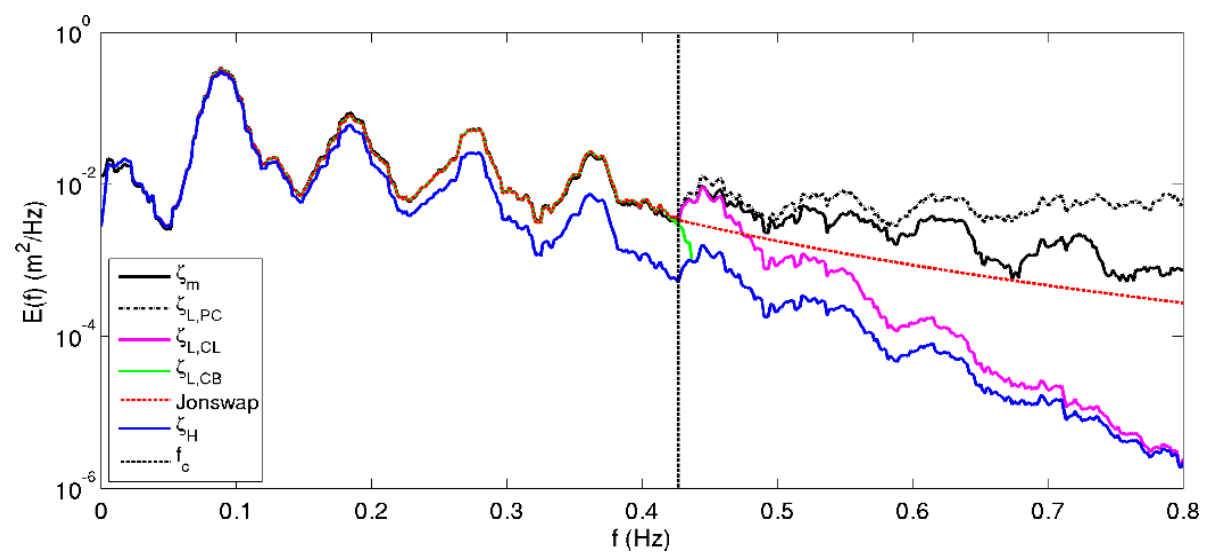

Figure 3. Spectres de densité d'énergie, $E(f)$, de la surface libre mesurée $\left(\zeta_{m}\right)$ et par la reconstruction $H\left(\zeta_{H}\right), L P C\left(\zeta_{L, P C}\right), L C L\left(\zeta_{L, C L}\right), L C B\left(\zeta_{L, C B}\right)$ et de JONSWAP $(n=4)$.

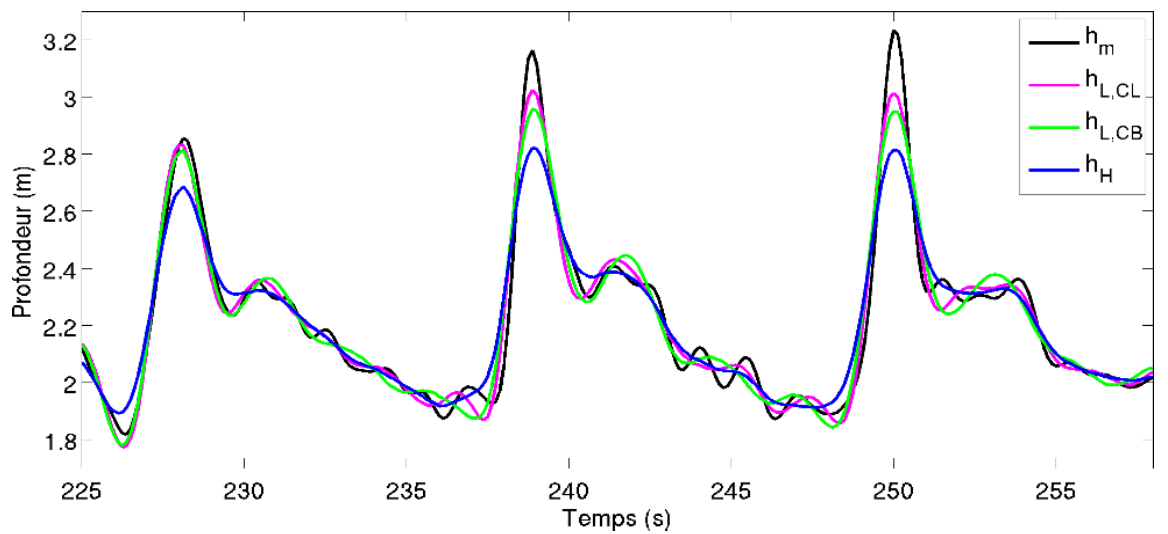

Figure 4. Profondeur d'eau mesurée $\left(h_{m}\right)$ et calculée par la reconstruction $H\left(h_{H}\right), L P C$ $\left(h_{L, P C}\right), L C L\left(h_{L, C L}\right), L C B\left(h_{L, C B}\right)$. Zoom sur un groupe de vagues.

Tableau 1 : Erreur sur la hauteur significative et sur la plus grosse vague.

\begin{tabular}{lllllll}
\hline & $H$ & $L C B$ & $L C L$ & $S L$ & $S N L$ & Jonswap $(n=4)$ \\
\hline Erreur sur $H_{s}(\%)$ & 14,6 & 4,6 & 3,7 & 6,3 & 4,2 & 3,2 \\
\hline Erreur sur la plus grosse vague (\%) & 42,7 & 28,9 & 22,5 & 26,4 & 7,7 & - \\
\hline
\end{tabular}

\subsection{Reconstructions en eau peu profonde}

En eau peu profonde, les vagues deviennent très non-linéaires $(\varepsilon>>1)$ et faiblement dispersives $(\mu<<1)$. BONNETON et al. (2018) ont dérivé des formules permettant une reconstruction linéaire et non-linéaire faiblement dispersive (SL et SNL respectivement)

$$
\begin{aligned}
& \zeta_{\mathrm{SL}}=\zeta_{\mathrm{H}}-\frac{\mathrm{h}_{0}}{2 \mathrm{~g}}\left(1-\left(\frac{\delta_{\mathrm{m}}}{\mathrm{h}_{0}}\right)^{2}\right) \partial_{\mathrm{t}}^{2} \zeta_{\mathrm{H}} \\
& \zeta_{\mathrm{SNL}}=\zeta_{\mathrm{SL}}-\frac{1}{\mathrm{~g}}\left(\partial_{\mathrm{t}}\left(\zeta_{\mathrm{SL}} \partial_{\mathrm{t}} \zeta_{\mathrm{SL}}\right)-\left(\frac{\delta_{\mathrm{m}}}{\mathrm{h}_{0}}\right)^{2}\left(\partial_{\mathrm{t}} \zeta_{\mathrm{SL}}\right)^{2}\right)
\end{aligned}
$$

Contrairement à la reconstruction linéaire classique (équation (2)), les équations (7) et (8) sont applicables localement en temps et ne nécessitent pas l'utilisation de la relation 


\section{Thème 1 - Hydrodynamique côtière}

de dispersion et donc de fréquence de coupure. Cependant, pour calculer les dérivées temporelles dans les équations (7) et (8), il est nécessaire d'introduire une fréquence de coupure $f_{c}$ pour éliminer le bruit de la mesure. Il est important de noter que cette fréquence de coupure est beaucoup moins restrictive $\left(f_{c}=1 \mathrm{~Hz}\right)$ que celle utilisée pour la méthode linéaire classique.

\section{Résultats}

\subsection{Comparaison des reconstructions linéaires et non-linéaires}

Comme attendu, la reconstruction non-linéaire en faible profondeur (SNL) permet de très bien reproduire la hauteur des plus grosses vagues ainsi que leur forme asymétrique (voir figure 5 et le tableau 1). La reconstruction linéaire en faible profondeur (SL) améliore légèrement la hauteur des vagues et leur forme par rapport au linéaire classiquement utilisée (L). De plus, les reconstructions faiblement dispersives conduisent à une très bonne estimation de l'énergie. Celles-ci reproduisent bien le pic des harmoniques secondaires (voir figure 6) contrairement à la méthode linéaire classique qui génère des oscillations parasites entre les vagues (voir figure 5).

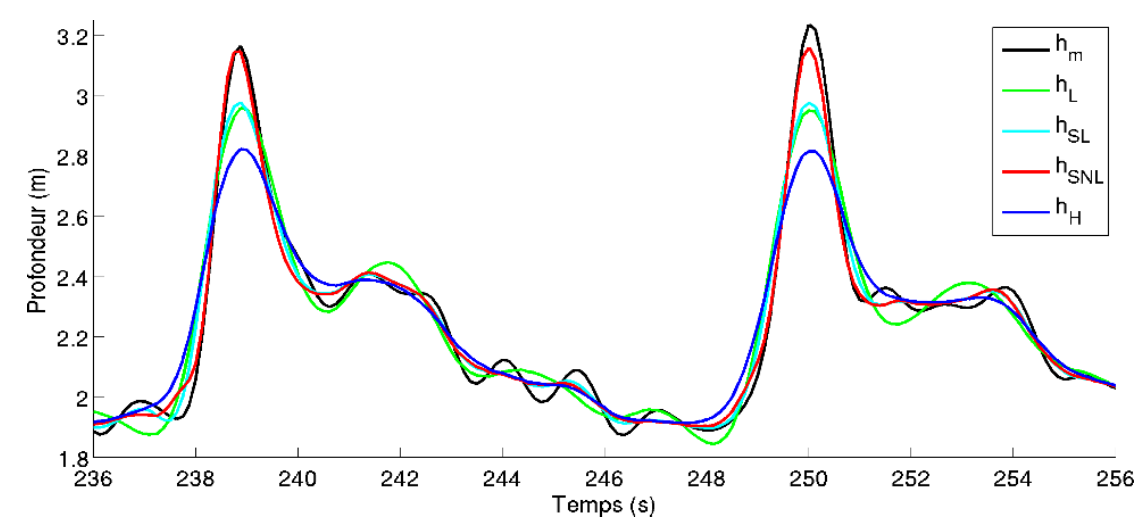

Figure 5. Profondeur d'eau mesurée $\left(h_{m}\right)$ et calculée par la reconstruction $H\left(h_{H}\right), L$ $\left(h_{L}\right)$, $S L\left(h_{S L}\right)$, SNL $\left(h_{S N L}\right)$. Zoom sur un groupe de vagues.

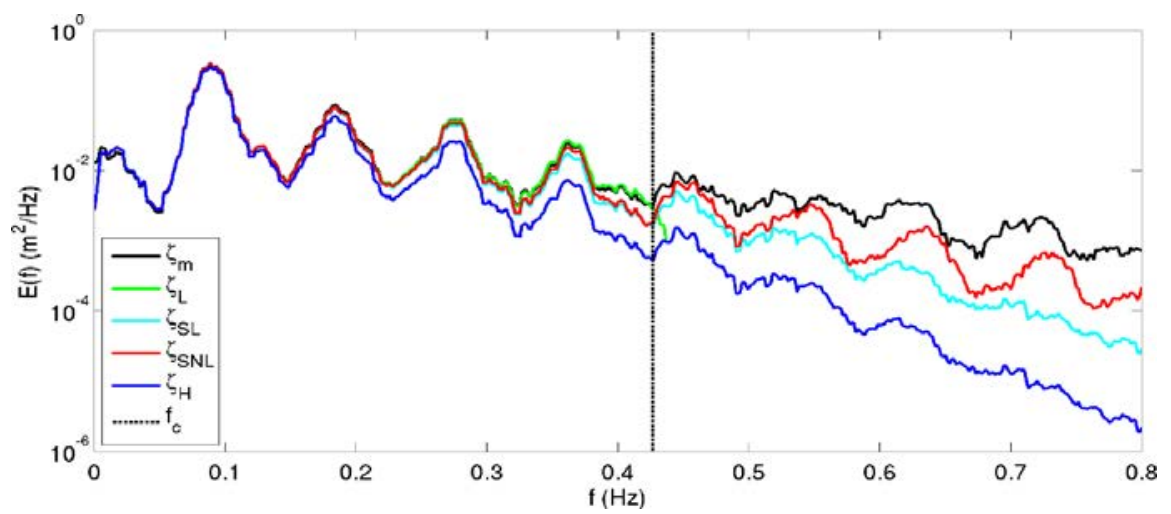

Figure 6. Spectres de densité d'énergie, $E(f)$, de la surface libre mesurée $\left(\zeta_{m}\right)$ et calculée par la reconstruction $H\left(\zeta_{H}\right), L\left(\zeta_{L}\right), S L\left(\zeta_{S L}\right), S N L\left(\zeta_{S N L}\right)$. 


\section{XVèmes Journées Nationales Génie Côtier - Génie Civil \\ La Rochelle, 29 au 31 mai 2018}

\subsection{Analyse vague à vague}

On effectue une analyse vague à vague sur une fenêtre de $20 \mathrm{~min}$ avant le déferlement. En eau peu profonde, la présence de mouvements basse fréquence peut conduire à des crêtes en dessous du niveau d'eau moyen et des creux au-dessus du niveau d'eau moyen. L'analyse zero-crossing, identifiant chaque vague lors d'un passage par le niveau d'eau moyen, n'est donc pas applicable puisque certaines vagues ne seront pas détectées. On choisit donc d'identifier les crêtes et les creux de chaque vague lors d'un changement de pente. Pour éviter de détecter les petites oscillations, on impose une hauteur minimale crête à creux de $10 \mathrm{~cm}$ et une période minimale entre 2 crêtes consécutives d'un quart de la période de pic (similaire à POWER et al., 2010). La présence de mer de vent rend difficile la détection précise des creux. On travaille donc sur l'élévation des crêtes de chaque vague et non sur la hauteur (voir figure 7).

La reconstruction non-linéaire faiblement dispersive estime très bien l'élévation des crêtes de toutes les vagues $\left(\mathrm{r}^{2}=0,97\right)$ contrairement à la reconstruction linéaire faiblement dispersive qui sous-estime les vagues les plus énergétiques (voir figure 7).

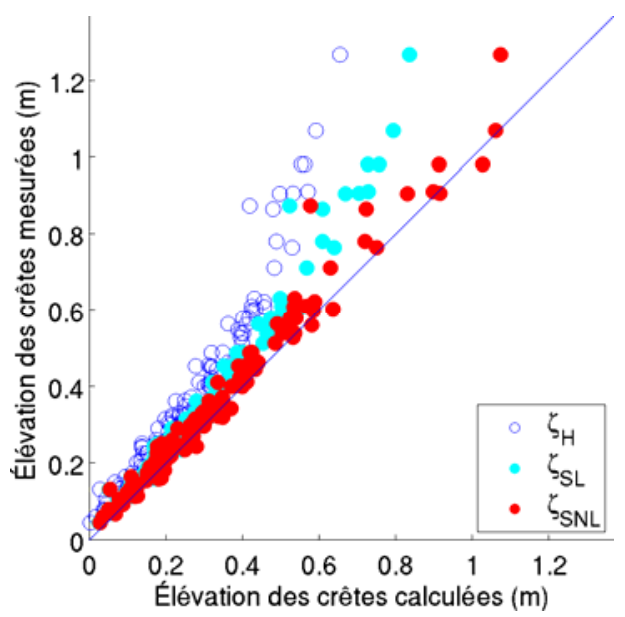

Figure 7. Comparaison des élévations des crêtes mesurées et calculées par la reconstruction $H$, SL et SNL.

\section{Conclusions}

En eau peu profonde, la théorie linéaire sous-estime largement la hauteur des plus grosses vagues. Les méthodes empiriques associées à la reconstruction linéaire améliorent légèrement la hauteur significative mais sous-estiment encore très fortement les plus grosses vagues. La reconstruction non-linéaire faiblement dispersive de BONNETON et al. (2018) diminue l'erreur de la hauteur de la plus grosse vague de $37 \%$ par rapport à la reconstruction hydrostatique. Elle décrit aussi très bien l'asymétrie des vagues et la densité d'énergie de la surface libre autour des harmoniques fondamentaux et secondaires. Ceci montre qu'une approche non-linéaire est essentielle pour la reconstruction du champ de vagues en zone de levée et proche du point de 


\section{Thème 1 - Hydrodynamique côtière}

déferlement. Pour des vagues se propageant en profondeur intermédiaire, une approche non-linéaire entièrement dispersive est requise (BONNETON \& LANNES, 2017). En plus des données de pression, la mesure de la vitesse permettrait une reconstruction entièrement non-linéaire et de prendre en compte la présence d'un courant moyen (courant de marée ou circulation induite par les vagues). Ces travaux pourraient constituer une nouvelle approche dans l'étude des vagues non-linéaires.

Remerciements : La thèse d'Arthur Mouragues a été cofinancée par une bourse DGARégion Nouvelle Aquitaine. Cette étude a reçu un financement du projet Région Nouvelle Aquitaine contrat n²017-1R20107.

\section{Références bibliographiques}

BISHOP C.T., DONELAN M.A. (1987). Measuring waves with pressure transducers. Coastal Engineering, 11 (4), 309-328. https://doi.org/10.1016/0378-3839(89)90014-8

BONNETON P., LANNES D. (2017). Recovering water wave elevation from pressure measurements. Journal of Fluid Mechanics, 833, 399-429. https://doi.org/10.1017/jfm.2017.666 BONNETON P., LANNES D., MARTINS K., MICHALLET H. (2018). A nonlinear weakly dispersive method for recovering the surface wave elevation from pressure measurements (submitted to Coastal Engineering).

DUBARBIER B., CASTELLE B., MARIEU V., RUESSINK G. (2015). Process-based modeling of cross-shore sandbar behavior. Coastal Engineering, 95, 35-50.

https://doi.org/10.1016/j.coastaleng.2014.09.004

GREEN M.O. (1999). Test of sediment initial-motion theories using irregular-wave field data. Sedimentology, 46, 427-441. https://doi.org/10.1046/j.1365-3091.1999.00221.x

GUZA R.T., THORNTON E.B. (1980). Local and shoaled comparisons of sea surface elevations, pressures and velocities. Journal of Geophysical Research, 85(C3), 15241530. https://doi.org/10.1029/JC085iC03p01524

KARIMPOUR A., CHEN Q. (2017). Wind wave analysis in depth limited water using OCEANLYZ, A MATLAB toolbox. Computers \& Geosciences 106, 181-189.

10.1016/j.cageo.2017.06.010

MARTINS K., BLENKINSOPP C.E., ALMAR R., ZANG J. (2017). The influence of swash-based reflection on surf zone hydrodynamics: a wave-by-wave approach. Coastal Engineering, 122, 27-43. https://doi.org/10.1016/j.coastaleng.2017.01.006

NEUMEIER U. neumeier.perso.ch.

POWER H.E., HUGHES M.G., AAGAARD T., BALDOCK T.E. (2010). Nearshore wave height variation in unsaturated surf. Journal of Geophysical Research : Oceans, 115 (C8). https://doi.org/10.1029/2009JC005758

TSAI C.H., HUANG M.C., YOUNG F.J., LIN Y.C., LI H.W. (2005). On the recovery of surface wave by pressure transfer function. Ocean Engineering, 32(10), 1247-1259. https://doi.org/10.1017/jfm.2017.666 